\title{
Quantitative Contribution of Six Major Transporters to the Hepatic Uptake of Drugs: "SLC-Phenotyping" Using Primary Human Hepatocytes ${ }^{[}$
}

\author{
Yi-an Bi, Chester Costales, Sumathy Mathialagan, Mark West, Soraya Eatemadpour, \\ Sarah Lazzaro, Laurie Tylaska, ${ }^{1}$ Renato J. Scialis, ${ }^{2}$ Hui Zhang, John Umland, (1) Emi Kimoto, \\ David A. Tess, Bo Feng, ${ }^{3}$ Larry M. Tremaine, ${ }^{4}$ (1) Manthena V. S. Varma, \\ and A. David Rodrigues
}

Medicine Design, Worldwide Research and Development, Pfizer Inc., Groton, Connecticut

Received February 19, 2019; accepted April 8, 2019

\begin{abstract}
Hepatic uptake transporters [solute carriers (SLCs)], including organic anion transporting polypeptide (OATP) 1B1, OATP1B3, OATP2B1, sodium-dependent taurocholate cotransporting polypeptide (NTCP), and organic anion (OAT2) and organic cation (OCT1) transporters, play a key role in determining the systemic and liver exposure of chemically diverse drugs. Here, we established a phenotyping approach to quantify the contribution of the six SLCs, and passive diffusion, to the overall uptake using plated human hepatocytes (PHHs). First, selective inhibitor conditions were identified by screening about 20 inhibitors across the six SLCs using single-transfected human embryonic kidney 293 cells. Data implied rifamycin SV $(20 \mu \mathrm{M})$ inhibits three OATPs, while rifampicin $(5 \mu \mathrm{M})$ inhibits OATP1B1/1B3 only. Further, hepatitis B virus myristoylated-preS1 peptide $(0.1 \mu \mathrm{M})$, quinidine $(100 \mu \mathrm{M})$, and ketoprofen $(100-300 \mu \mathrm{M})$ are relatively selective against
\end{abstract}

NTCP, OCT1, and OAT2, respectively. Second, using these inhibitory conditions, the fraction transported $\left(f_{t}\right)$ by the individual SLCs was characterized for 20 substrates with PHH. Generally, extended clearance classification system class 1A/3A (e.g., warfarin) and 1B/3B compounds (e.g., statins) showed predominant OAT2 and OATP1B1/1B3 contribution, respectively. OCT1mediated uptake was prominent for class $2 / 4$ compounds (e.g., metformin). Third, in vitro $f_{t}$ values were corrected using quantitative proteomics data to obtain "scaled $f_{t}$." Fourth, in vitro-in vivo extrapolation of the scaled OATP1B1/1B3 $f_{t}$ was assessed, leveraging statin clinical drug-drug interaction data with rifampicin as the perpetrator. Finally, we outlined a novel stepwise strategy to implement phenotypic characterization of SLC-mediated hepatic uptake for new molecular entities and drugs in a drug discovery and development setting.

\section{Introduction}

Hepatic (sinusoidal) uptake is the first step and is often considered as rate-determining in the hepatic clearance of drugs (Shitara et al., 2013; Varma et al., 2015). Such uptake transport is mediated by a cadre of solute carriers (SLCs) comprising the organic anion transporting polypeptides (OATP1B1, OATP1B3, and OATP2B1; SLCO1B1, SLCO1B3,

All authors are full-time employees of Pfizer Inc. The authors have no conflicts of interest that are directly relevant to this study.

${ }^{1}$ Current affiliation: Cybrexa Therapeutics, New Haven, CT.

${ }^{2}$ Current affiliation: Metabolism and Pharmacokinetics, Preclinical Candidate Organization, Bristol-Myers Squibb, Princeton, NJ.

${ }^{3}$ Current affiliation: Drug Metabolism and Pharmacokinetics Group, Vertext Pharmaceutics, Boston, MA.

${ }^{4}$ Current affiliation: Tremaine DMPK Consulting, LLC, Merritt Island, FL https://doi.org/10.1124/jpet.119.257600.

S This article has supplemental material available at jpet.aspetjournals.org. and SLCO2B1), organic anion transporter 2 (OAT2, SLC22A7), organic cation transporter 1 (OCT1, SLC22A1), and sodiumdependent taurocholate cotransporting polypeptide (NTCP, SLC10A1) (Giacomini et al., 2010; Zamek-Gliszczynski et al., 2018). Importantly, the expression and function of these transporters are modulated by genotype, disease, drug-drug interactions (DDIs), and age (König et al., 2013; Elmorsi et al., 2016; Zhou et al., 2017). Moreover, liver SLCs are often paired with drug-metabolizing enzymes with regard to drug disposition, commonly referred to as "transporter-enzyme interplay," which also serve as the loci of DDI and whose expression function is also impacted by genotype, age, and disease (Shitara et al., 2006, 2013; Li et al., 2014; Varma et al., 2015). Therefore, it is important to quantitatively understand the major transporter(s) involved in the uptake clearance of a substrate drug (vs. passive uptake clearance) and leverage the data to support in vitro-in vivo extrapolations-specifically,

ABBREVIATIONS: AUC, area under the plasma concentration-time curve; BCRP, breast cancer resistance protein; CCK8, cholecystokinin octapeptide; DDI, drug-drug interaction; ECCS, extended clearance classification system; E17G, estradiol 17 $\beta$-glucuronide; E3S, estrone 3-sulfate; HBSS, Hanks' balanced salt solution; HBVpep, hepatitis B virus myristoylated-preS1 peptide; HEK293, human embryonic kidney 293; IVIV, in vitro-in vivo; LC-MS/MS, liquid chromatography-tandem mass spectrometry; MW, molecular weight; NME, new molecular entity; NTCP, sodiumdependent taurocholate cotransporting polypeptide; OAT, organic anion transporter; OATP, organic anion-transporting polypeptide; OCT, organic cation transporter; $\mathrm{PHH}$, plated human hepatocyte; REF, relative expression factor; R3G, resveratrol-3-O-glucuronide; SLC, solute carrier. 
determine the fraction of total hepatocyte uptake mediated by each individual transporter $\left(f_{t}\right)$ (i.e., $f_{t, O A T P}+f_{t, N T C P}+$ $f_{t, \text { OCT1 }}+f_{t, \text { OAT2 }}+f_{t, \text { passive }} \sim 1$ ). By analogy with cytochrome $\mathrm{P} 450$ reaction phenotyping, such an exercise can be referred to as "SLC-phenotyping" (Williams et al., 2005).

Distinct transporter activity has been shown for drugs in different classes of the extended clearance classification system (ECCS) - a framework to predict clearance mechanism based on compound ionization state, molecular weight (MW), and in vitro membrane permeability (Varma et al., 2015; El-Kattan and Varma, 2018). Clearly, hepatic clearance of high-MW acids/zwitterions (ECCS class 1B/3B; e.g., statins) is associated with OATP1B1/1B3-mediated hepatic uptake (Shitara et al., 2013; Varma et al., 2015, 2017b). Results of our recent studies have shown that the majority of ECCS class 1A drugs (low-MW acids/zwitterions; e.g., warfarin, tolbutamide) are substrates to OAT2 (Bi et al., 2018b; Kimoto et al., 2018). Similarly, ECCS class 4 drugs (low-permeability bases/neutrals; e.g., metformin) potentially involve OCT1 in their hepatic disposition (El-Kattan and Varma, 2018). Given the importance of uptake transporters, it has become increasingly common practice to assess new molecular entities (NMEs) as liver transporter substrates in vitro using various combinations of human SLC-transfected cells and primary human hepatocytes. For the latter, studies have suggested that one can differentiate total active uptake (vs. passive) by incubating a high concentration of a pan-SLC inhibitor, such as rifamycin SV ( 1 $\mathrm{mM})$, or comparing uptake rates at $37^{\circ} \mathrm{C}$ versus $4^{\circ} \mathrm{C}$ (Bi et al., 2017; Chothe et al., 2018). However, due to the limitation of selective inhibitors, assessing individual SLC activity in human hepatocytes has proven to be challenging. Undoubtedly, the OATPs are the most studied human liver SLCs, and researchers have attempted relative activity factor, relative expression factor (REF), and small interfering RNA approaches to assess their contribution in the hepatic uptake of substrate drugs (Williamson et al., 2013; Kunze et al., 2014; Mitra et al., 2018). In comparison, the tools to assess other SLCs (and their contributions) in human hepatocytes are not well developed. For example, NTCP activity is often determined by incubating hepatocytes in sodium-free (vs. sodium-containing) medium, and no selective inhibitor has been identified (Bi et al., 2017). Likewise, there are only a few examples describing OAT2 and OCT1 substrate phenotyping with human hepatocytes (De Bruyn et al., 2011; Bi et al., 2018a; Mathialagan et al., 2018).

In this study, about 20 compounds were screened as inhibitors across six major uptake transporters [individually expressed in human embryonic kidney 293 (HEK293) cells] in an attempt to identify, characterize, and validate selective inhibitor conditions for SLC-phenotyping using plated human hepatocytes (PHHs). The fraction transported $\left(f_{t}\right)$ by individual transporters and passive diffusion was then determined via $\mathrm{PHH}$ incubations and corrected for the protein expression differences between cryopreserved hepatocytes and liver samples for a set of 20 diverse substrates/drugs representing various ECCS classes: estradiol $17 \beta$-glucuronide (E17G), estrone 3 -sulfate (E3S), cholecystokinin octapeptide (CCK8), resveratrol-3-O-glucuronide (R3G), taurocholic acid, cyclic $3^{\prime}, 5^{\prime}$-guanosine monophosphate (cGMP), rosuvastatin, pitavastatin, pravastatin, fluvastatin, bromfenac, entacapone, fluorescein, tolbutamide, meloxicam, R/S-warfarin, metformin, ranitidine, and thiamine. Finally, we assessed in vitro-in vivo (IVIV) extrapolation of $f_{t, O A T P 1 B 1 / 1 B 3}$, leveraging clinical statin DDI data following a single dose of rifampicin, a probe OATP1B1/1B3 inhibitor.

\section{Materials and Methods}

Chemicals and Reagents. Cyclosporine A, ketoprofen, meloxicam, quinidine, ranitidine, rifampicin, rifamycin SV, taurocholate acid, and tolbutamide were purchased from Sigma-Aldrich (St. Louis, MO). Statins were purchased from Sequoia Research Products Ltd. (Oxford, UK). All other compounds were obtained from the Pfizer Chemical inventory system. $\left[{ }^{3} \mathrm{H}\right]$-Metformin and $\left[{ }^{3} \mathrm{H}\right]$-thiamine were purchased from American Radiolabeled Chemicals Inc. (St. Louis, MO). $\left[{ }^{3} \mathrm{H}\right]$-Taurocholate acid, $\left[{ }^{3} \mathrm{H}\right]$-cGMP, CCK8, E17G, and E3S were purchased from PerkinElmer Life Sciences (Boston, MA). Hepatitis B virus myristoylated-preS1 peptide (HBVpep) was synthesized by New England Peptide (Gardner, MA); the amino acid sequence was derived from the preS1 region of HBV (D-type, GenBank accession number U9555.1) containing residues $2-48$ and modified with $\mathrm{N}$-terminal myristoylation (König et al., 2014; Yan et al., 2014). InVitroGro-HT, -CP, and -HI hepatocyte media were purchased from Celsis IVT (Baltimore, MD). Collagen I-coated 24-well plates were obtained from BD Biosciences (Franklin Lakes, NJ). Cryopreserved human hepatocytes lot Hu8246 (female, Caucasian, 37 years old, no medication, two to three alcoholic drinks per week, nonreactive to hepatitis $\mathrm{B} / \mathrm{C}$ and human immunodeficiency virus) were purchased from Life Technologies (Thermo Fisher Scientific, Carlsbad, CA). NP40 protein lysis buffer was purchased from Thermo Fisher (Franklin, MA). HEK293 cells stably transfected with human OATP1B1, OATP1B3, or OATP2B1 were generated at Pfizer Inc. (Sandwich, UK). HEK293 cells stably transfected with human NTCP, OCT1, and OAT2(tv-1) were obtained from the laboratories of Per Artursson (Uppsala University, Sweden), Kathleen Giacomini (University of California), and Ryan Pelis (Dalhousie University, Canada), respectively. Dulbecco's modified Eagle's medium, fetal bovine serum, nonessential amino acids, GlutaMAX-1, sodium pyruvate, penicillin, and streptomycin solution were obtained from Invitrogen (Carlsbad, CA).

In Vitro Transport Studies Using Transporter-Transfected Cells. HEK293 cells, wild-type and stably transfected with NTCP, OATP1B1, OATP1B3, OATP2B1, OAT2, and OCT1, were seeded at a density of $0.5-1.2 \times 10^{5}$ cells/well on BioCoat 48- or 96-well poly-Dlysine-coated plates (Corning Inc., Corning, NY) and grown in Dulbecco's modified Eagle's medium containing 10\% FBS and 1\% sodium pyruvate for 48 hours at $37^{\circ} \mathrm{C}, 90 \%$ relative humidity, and $5 \%$ $\mathrm{CO}_{2}$. OATP1B1-, OATP1B3-, OATP2B1-, and NTCP-HEK293 cells were supplemented with non-essential amino acid and GlutaMAX. OCT1- and OAT2-HEK293 cells were supplemented with $1 \%$ gentamycin, $1 \%$ sodium pyruvate, and $50 \mu \mathrm{g} / \mathrm{ml}$ hygromycin $\mathrm{B}$.

For the inhibition studies, HEK293 cells were washed three times with warm uptake buffer [Hanks' balanced salt solution (HBSS) with $20 \mathrm{mM}$ HEPES, $\mathrm{pH}$ 7.4] followed by incubation with test compounds containing probe substrates: $0.5 \mu \mathrm{M}\left[{ }^{3} \mathrm{H}\right]$-cGMP (OAT2), $10 \mu \mathrm{M}\left[{ }^{14} \mathrm{C}\right]$ metformin (OCT1), $10 \mu \mathrm{M}\left[{ }^{3} \mathrm{H}\right]$-taurocholic acid (NTCP), or $5 \mu \mathrm{M}$ rosuvastatin (OATP1B1/1B3/2B1) (Bi et al., 2017, 2018a). For substrate studies, test compounds were incubated for 2-4 minutes. Cellular uptake was terminated by washing the cells four times with ice-cold transport buffer and then lysing with $0.2 \mathrm{ml}$ of $1 \% \mathrm{NP} 40$ in water or methanol containing internal standard. Radioactivity in each sample was quantified by measurement on a Wallac liquid scintillation counter (PerkinElmer Life Sciences) for radiolabeled compounds or by a liquid chromatography-tandem mass spectrometry (LC-MS/ MS) system. The total cellular protein content was determined by using a Pierce BCA Protein Assay kit according to the manufacturer's specifications. Uptake ratio was then derived as a ratio of 
accumulation in transfected cells to accumulation in wild-type cells ( $\mathrm{Bi}$ et al., 2018a; Mathialagan et al., 2018).

Uptake and Inhibition Studies Using Cryopreserved PHH. The hepatic uptake assay was performed using short-term culture primary human hepatocytes as described previously, with some modification (Bi et al., 2017). In brief, cryopreserved hepatocytes were thawed at $37^{\circ} \mathrm{C}$ and seeded into 24 -well collagen I-coated plates with $0.35 \times 10^{6}$ cells/well in a volume of $0.5 \mathrm{ml} /$ well. The cells were cultured in the InVitroGro-CP medium overnight ( 18 hours). Functional studies using a variety of probe substrates, comparing a previously validated 6-hour culture time (Bi et al., 2017) versus 18-hour culture time, suggested lack of functional difference under these conditions (data not shown). Cell culture was preincubated for 10 minutes at $37^{\circ} \mathrm{C}$ with HBSS with or without inhibitors. The preincubation buffer was aspirated, and the uptake and inhibition reactions were initiated by addition of prewarmed buffer containing test compounds with or without inhibitors. The reactions were terminated at designated time points $(0.5,1,2,5$ minutes) by adding ice-cold HBSS immediately after removal of the incubation buffer. The cells were washed three times with ice-cold HBSS and lysed with $100 \%$ methanol containing internal standard or $0.5 \%$ Triton-100 for radiolabeled compound, and the samples were analyzed by LC-MS/MS (Supplemental Methods) or by liquid scintillation counter. Uptake rates were estimated from the initial time course (0.5-2 minutes) by linear regression (Bi et al., 2017, 2018a).

LC-MS/MS Method. LC-MS/MS analyses were performed on a SCIEX Triple Quad 6500 mass spectrometer (SCIEX, Ontario, Canada) equipped with a TurboIonSpray interface. The highperformance liquid chromatography systems consisted of an Agilent 1290 Infinity binary pump and ADDA autosampler (Agilent Technologies, Santa Clara, CA). All instruments were controlled and synchronized by SCIEX Analyst software (version 1.6.2) working in tandem with the ADDA software. Mobile phases were $0.1 \%$ formic acid in water (mobile phase A) and $0.1 \%$ formic acid in acetonitrile (mobile phase B). The chromatographic separation was carried out on a Phenomenex Kinetex C18 $100 \AA 30 \times 2.1-\mathrm{mm}$ column with a flow rate of $0.8 \mathrm{ml} / \mathrm{min}$. The injection volume was $10 \mu \mathrm{l}$. The following gradient was used to elute samples: $5 \%$ solvent $\mathrm{B}$ for 0.2 minutes, increasing to $95 \% \mathrm{~B}$ for 0.5 minutes, held at $95 \%$ $\mathrm{B}$ for 0.3 minutes, reduction to $5 \% \mathrm{~B}$ over 0.02 minutes, and held at $5 \%$ solvent B for 0.48 minutes (total run time 1.5 minutes). For the mass spectrometer, the TurboIonSpray interface was operated in the positive/negative switching ion mode at 5000/-4500 V and $600^{\circ} \mathrm{C}$. Quadrupoles Q1 and Q3 were set on unit resolution. Multiplereaction monitoring mode using specific precursor/product ion transitions was used for quantification. Linear regression was fitted to data of standard solutions using $1 / \mathrm{Y}^{2}$ weighting. Data processing was performed using MultiQuant software (version 3.0.2; Sciex).

Data Analysis. Uptake clearance from $\mathrm{PHH}$ incubations was estimated from the initial time course $(0.5-2$ minutes) by linear regression (Bi et al., 2017). Fraction transported $\left(f_{t}\right)$ by transporter(s)specific active transport or passive diffusion is defined from uptake clearance in the absence (control) and presence of inhibitors by eqs. 1 and 2 , respectively:

$$
\begin{aligned}
f_{t, \text { active }} & =1-\frac{\text { uptake }_{\text {clearance }} \text { inhibitor }}{\text { uptake clearance }_{\text {control }}} \\
f_{t, \text { passive }} & =\frac{\text { uptake clearance }_{\text {inhibitor }}}{\text { uptake clearance }_{\text {control }}}
\end{aligned}
$$

Scaled fraction transported ("scaled $f_{t}$ ") is defined by the following equation:

$$
\begin{aligned}
& \text { scaled total transport }=f_{t, p a s s i v e}+f_{t, O A T P 1 B} \cdot \mathrm{REF}_{O A T P 1 B} \\
& \quad+f_{t, O A T P 2 B 1} \cdot \mathrm{REF}_{O A T P 2 B 1}+f_{t, N T C P} \cdot \mathrm{REF}_{N T C P} \\
& \quad+f_{t, O A T 2} \cdot \mathrm{REF}_{O A T 2}+f_{t, O C T 1} \cdot \mathrm{REF}_{O C T 1} .
\end{aligned}
$$

$\mathrm{REF}$ is defined as the ratio of transporter protein expression in human liver samples to that in cryopreserved human hepatocytes (Shitara et al., 2013; Zamek-Gliszczynski et al., 2013; Li et al., 2014). REF values were estimated using our previously published quantitative proteomics data (LC-MS-based approach)-mean of protein abundance in multiple liver samples versus multiple single-donor hepatocyte lots. The hepatocyte lot used for functional studies here closely represented $( \pm 30 \%)$ population mean protein abundance for OATPs (unpublished data) and OAT2/7s (Vildhede et al., 2018). REF values for OATP1B, OATP2B1, NTCP, OAT2, and OCT1 are 2.83 (average of OATP1B1 and OATP1B3) (Kimoto et al., 2012), 2.36 (Kimoto et al., 2012), 1.06 (Qiu et al., 2013), 1.78 (Vildhede et al., 2018), and 1.02 (unpublished data), respectively.

Finally, scaled $f_{t}$ via specific transporter or passive diffusion is described as follows (OATP1B as representative):

$$
\text { scaled } f_{t, O A T P 1 B}=\frac{f_{t, O A T P 1 B} \cdot \mathrm{REF}_{O A T P 1 B}}{\text { scaled total transport }}
$$

Static Model Predictions of Statin Area under the Curve Ratio Using $\boldsymbol{f}_{\boldsymbol{t}}$. The area under the plasma concentration-time curve (AUC) ratio of an oral victim drug, in the presence $\left(\mathrm{AUC}_{p o}^{\prime}\right)$ and absence $\left(\mathrm{AUC}_{p o}\right)$ of perpetrator, can be described by the following equations (Varma et al., 2014):

$$
\begin{aligned}
\mathrm{AUC} \text { ratio } & =\frac{\mathrm{AUC}_{p o}^{\prime}}{\mathrm{AUC}_{p o}}=\frac{F a^{\prime}}{F a} \cdot \frac{F g^{\prime}}{F g} \cdot \frac{F h^{\prime}}{F h} \cdot \frac{\left(\mathrm{CL}_{h}+\mathrm{CL}_{r}\right)}{\left(\mathrm{CL}_{h}{ }^{\prime}+\mathrm{CL}_{r}{ }^{\prime}\right)} \\
F h & =1-\frac{\mathrm{CL}_{h}}{Q_{h}} \text { and } F h^{\prime}=1-\frac{\mathrm{CL}_{h}{ }^{\prime}}{Q_{h}}
\end{aligned}
$$

where $F a, F g$, and $F h$ represent the fraction of drug absorbed, fraction of drug escaping hepatic extraction, respectively. $F a^{\prime}, F g^{\prime}$, and $F h^{\prime}$ are corresponding parameters in the presence of perpetrator. $\mathrm{CL}_{h}, \mathrm{CL}_{r}$, $\mathrm{CL}_{h}{ }^{\prime}$, and $\mathrm{CL}_{r}{ }^{\prime}$ represent hepatic and renal blood clearance in the absence and presence of the perpetrator, respectively:

$$
\begin{gathered}
\mathrm{CL}_{h}=\frac{Q_{h} \cdot f_{u, b l o o d} \cdot \mathrm{CL}_{\text {int }, h}}{Q_{h}+f_{u, b l o o d} \cdot \mathrm{CL}_{\text {int }, h}} \text { and } \mathrm{CL}_{h}{ }^{\prime}=\frac{Q_{h} \cdot f_{u, b l o o d} \cdot \mathrm{CL}_{\text {int }, h^{\prime}}{ }^{\prime}}{Q_{h}+f_{u, b l o o d} \cdot \mathrm{CL}_{\text {int }, h^{\prime}}} \\
\mathrm{CL}_{\text {int }, h}=\frac{Q_{h} \cdot \mathrm{CL}_{h}}{f_{u, \text { blood }} \cdot\left(Q_{h}-\mathrm{CL}_{h}\right)} .
\end{gathered}
$$

$Q_{h}$ is hepatic blood flow [20.7 $\mathrm{ml} / \mathrm{min} / \mathrm{kg}$ (Kato et al., 2003)], and $f_{u, \text { blood }}$ is fraction unbound in blood. $\mathrm{CL}_{i n t, h}$ and $\mathrm{CL}_{\text {int }, h^{\prime}}$ are intrinsic hepatic clearance in the absence and presence of the perpetrator, respectively. Assuming uptake (active + passive) is the rate-determining process in the hepatic clearance of statins, $C_{\text {int, } h}$ ' can be defined as follows (eq. 9) (Li et al., 2014; Varma and El-Kattan, 2016):

$$
\mathrm{CL}_{i n t, h^{\prime}}{ }^{\prime}=\mathrm{CL}_{i n t, h} \cdot\left[\frac{\operatorname{scaled} f_{t, O A T P 1 B}}{\left(1+\frac{I_{u, m a x, i n}}{K_{i}}\right)}+\left(1-\operatorname{scaled} f_{t, O A T P 1 B}\right)\right] .
$$

"Scaled $f_{t, O A T P 1 B}$ " represents the fraction transported by OATP1B/ 1B3, measured in vitro in PHH and corrected for the REF. $K_{i}$ is the inhibition constant, and $I_{u, \max , \text { in }}$ is the maximum unbound perpetrator concentration at the inlet to liver.For interaction with rifampicin (600-mg single dose) as a perpetrator, we assumed no change in $F a$, $F g$, and $\mathrm{CL}_{r}$ of statins evaluated. All input values for statins, $\mathrm{CL}_{h}$ and $\mathrm{CL}_{r}$, were taken from Izumi et al. (2017) (rosuvastatin: $f_{u, \text { blood }}=0.298$, $\mathrm{CL}_{h}=16.45, \mathrm{CL}_{r}=6.37 \mathrm{ml} / \mathrm{min} / \mathrm{kg}$; pitavastatin: $f_{u, \text { blood }}=0.0118$, $\mathrm{CL}_{h}=13.95, \mathrm{CL}_{r}=0 \mathrm{ml} / \mathrm{min} / \mathrm{kg}$; pravastatin: $f_{u, \text { blood }}=0.888, \mathrm{CL}_{h}=$ $14.12, \mathrm{CL}_{r}=12.35 \mathrm{ml} / \mathrm{min} / \mathrm{kg}$; fluvastatin: $f_{u, b l o o d}=0.0073, \mathrm{CL}_{h}=$ 13.92, $\mathrm{CL}_{r}=0 \mathrm{ml} / \mathrm{min} / \mathrm{kg}$ ). Rifampicin $K_{i}$ and $I_{u, \max , \text { in }}$ (following 600 -mg single dose) values applied were $0.145 \mu \mathrm{M}$ [assuming $K_{i}=$ $\mathrm{IC}_{50} / 2$; OATP1B1 $\mathrm{IC}_{50} 0.29 \mu \mathrm{M}$ measured in the current study was used (Table 1)] and 1.875 $\mu \mathrm{M}$ (Varma et al., 2012), respectively. At this 
plasma unbound concentration, rifampicin selectively inhibits OATP1B1/OATP1B (see Results).

\section{Results}

In Vitro Inhibition of Six Hepatic Uptake Transporters in Transfected Cells. About 20 compounds were selected to screen for their inhibition potency against six known human liver SLCs based on prior published knowledge of their ability to inhibit one or more of these transporters (Fig. 1; Table 1). For instance, erlotinib is known to selectively inhibit OATP2B1 over other OATP isoforms, but its effect on non-OATPs is not clear (Karlgren et al., 2012). Well characterized probe substrates were used to measure the inhibition potency: rosuvastatin for OATP1B1/1B3/2B1, taurocholate for NTCP, metformin for OCT1, and cGMP for OAT2 (Giacomini et al., 2010; Brouwer et al., 2013; Mathialagan et al., 2018). OATP1B1-mediated rosuvastatin transport was inhibited by rifamycin SV, rifampicin, gefitinib, octreotide, sulfasalazine, and CCK8 with a clear concentration-dependent response. Erlotinib inhibited OATP2B1 and OCT1 with similar inhibition potency $\left(\mathrm{IC}_{50}\right)$, but inhibition was not apparent for other SLCs. Interestingly, OCT1 inhibitors also inhibited (e.g., erlotinib) or stimulated OATP2B1-mediated transport of rosuvastatin (e.g., 1-methyl-4-phenylpyridinium, TEA, prazosin, quinidine). Sulfated and nonsulfated CCK8 inhibited both OATP1B1 and OATP1B3 with similar potency but showed weaker OATP2B1 inhibition. Sulfasalazine showed relatively potent inhibition of OATP2B1 $\left(\mathrm{IC}_{50}\right.$ $\sim 1.7 \mu \mathrm{M})$ compared with OATP1B1/1B3 inhibition $\left(\mathrm{IC}_{50}\right.$ $\sim 25 \mu \mathrm{M})$. Both E3S and estropipate showed selective inhibition of OATP1B1 over OATP1B3 $\left(\mathrm{IC}_{50} \sim 0.2\right.$ vs. $\sim 300$ $\mu \mathrm{M})$. However, in replicate studies, we noted that their OATP1B1 inhibition curves were relatively flat and reached only $\sim 75 \%$ inhibition at maximum concentration tested $(300 \mu \mathrm{M})$. Moreover, estropipate also showed notable OATP2B1 inhibition $\left(\mathrm{IC}_{50} \sim 0.9 \mu \mathrm{M}\right)$.

Rifamycin SV at $20 \mu \mathrm{M}$ inhibited hepatic OATPs but had a minimal impact on the other SLCs (Table 2). Additionally, rifamycin SV inhibited all six SLCs in the concentration range studied (up to $1 \mathrm{mM}$ ). On the other hand, rifampicin at $10 \mu \mathrm{M}$ completely inhibited OATP1B1/1B3 but did not inhibit the other four SLCs, including OATP2B1. Finally, ketoprofen, quinidine, and HBVpep showed selective inhibition of OAT2, OCT1, and NTCP, respectively. Based on the collective results, the following inhibition conditions were implemented for SLC-phenotyping in PHHs (Table 2): rifampicin $(10 \mu \mathrm{M})$ for OATP1B1/1B3, rifamycin SV $(20 \mu \mathrm{M})$ for three OATPs, HBVpep $(0.1 \mu \mathrm{M})$ for NTCP, ketoprofen $(100-300 \mu \mathrm{M})$ for OAT2, and quinidine $(100 \mu \mathrm{M})$ for OCT1.

In Vitro Transport by Six SLCs in Transfected HEK293 Cells. A set of 20 compounds, spanning various ECCS classes, were assessed for their substrate affinity to the individual SLCs expressed in HEK293 cells (Table 3). Uptake ratio (accumulation in transfected cells to wild-type cells) of $>2$ was used as a criterion for transport activity. All ECCS class $1 \mathrm{~B}$ and $3 \mathrm{~B}$ compounds in the list (7 of 20 ) showed transport by OATPs. Additionally, many of these were also transported by NTCP. Taurocholate showed a much higher uptake ratio ( 185) with NTCP cells, although notable uptake was also apparent for OATP1B1/1B3 cells (uptake ratio 11-26). Similarly, E17G and CCK8 presented much higher uptake ratios with OATP1B1 (423) and OATP1B3 (371) cells, respectively. E3S was actively transported by all transporters except OCT1, although uptake was markedly high in the OATP1B1 and OATP2B1 cells. On the other hand, R3G showed transport by the three OATPs but with a higher uptake ratio $(\sim 54)$ in OATP2B1 cells. ECCS class 1A/3A compounds (cGMP, meloxicam, R/S-warfarin, and tolbutamide) were shown to be transported by OAT2 alone; however, bromfenac, entacapone, and fluorescein tested positive for OATP1B1 as well. Metformin, ranitidine, and thiamine (ECCS class 4) showed OCT1 activity.

In Vitro Uptake Phenotyping in Human Hepatocytes. Uptake of 20 compounds was also measured in PHHs in the absence and presence of the various chosen inhibitor conditions (Fig. 2). Based on the uptake activity in the presence of the various inhibitors and the REF of individual transporters, the scaled $f_{t}$ of individual transporter(s) and passive uptake was discerned (see Materials and Methods) (Fig. 2; Table 4). For E17G, hepatocyte uptake was completely inhibited in the presence of $10 \mu \mathrm{M}$ rifampicin and $1 \mathrm{mM}$ rifamycin SV. This in conjunction with substrate activity in transfected HEK293 cells indicated that $\mathrm{E} 17 \mathrm{G}$ is preferentially taken up by OATP1B1. Similarly, CCK8 uptake inhibition in the presence of $10 \mu \mathrm{M}$ rifampicin indicated preferential OATP1B1/1B3 uptake. Hepatocyte uptake of E3S was partially inhibited $(\sim 47 \%)$ in the presence of $10 \mu \mathrm{M}$ rifampicin and almost completely inhibited ( $\sim 93 \%)$ by $1 \mathrm{mM}$ rifamycin SV. Although E3S showed active uptake by OAT2 and NTCP in HEK293 cells, the contribution of these SLCs to hepatocyte uptake was not apparent, as ketoprofen and HBVpep showed no significant inhibition. R3G showed OATP2B1-specific uptake, with negligible inhibition by $10 \mu \mathrm{M}$ rifampicin. All OATP inhibitors (cyclosporine A, rifampicin, and rifamycin SV) significantly inhibited uptake of all statins tested, although the effects were relatively small for fluvastatin. Rosuvastatin and fluvastatin showed 15\%-20\% contribution by OATP2B1 and/or NTCP, while pitavastatin and pravastatin are primarily taken up by OATP1B1/1B3.

ECCS class 1A drugs meloxicam, R/S-warfarin, and tolbutamide were selectively inhibited by the OAT2 inhibitor, while class 4 drugs metformin and ranitidine showed selective OCT1 activity. Interestingly, thiamine showed almost equal contribution of OCT1-mediated active transport and passive diffusion. Generally, uptake of these compounds was not affected by OATP/NTCP inhibitor conditions (i.e., $10 \mu \mathrm{M}$ rifampicin, $20 \mu \mathrm{M}$ cyclosporine $\mathrm{A}, 0.1 \mu \mathrm{M}$ HBV peptide) but was inhibited by ketoprofen or quinidine and the pan-SLC inhibitor (1 mM rifamycin SV).

IVIV Extrapolation of Scaled $f_{t, O A T P 1 B 1 / 1 B 3}$ Using Statin-Rifampicin DDI Data. Clinical DDI data with single-dose 600-mg rifampicin was available for the statins as substrate drugs. At this dose, rifampicin plasma unbound $C_{\max }$ at the inlet of liver $\left(C_{\text {max }, \text { in }, u}\right)$ is about $1.87 \mu \mathrm{M}$-where only OATP1B1 and OATP1B3 are inhibited (Varma et al., 2012) (Fig. 1; Tables 1 and 2). Therefore, scaled $f_{t, O A T P 1 B 1 / 1 B 3}$ (measured via PHH incubations and corrected for REF) was used to predict the AUC change of the four statins following oral dosing with rifampicin. The static model (eqs. 5-9) considered a change in hepatic first-pass extraction and hepatic clearance of statins due to OATP1B1/1B3 inhibition and assumed no change in oral absorption, gut extraction, and renal clearance by rifampicin. Predicted AUC ratios are 


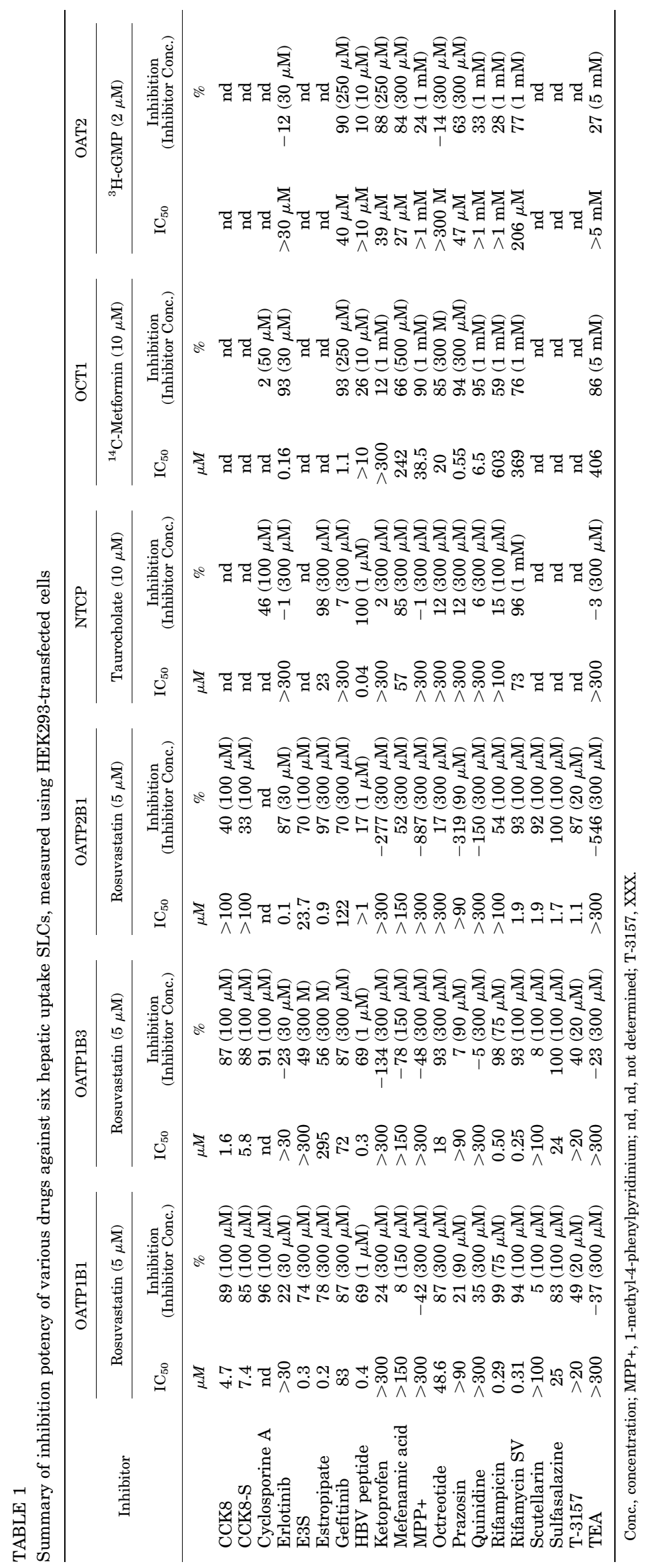



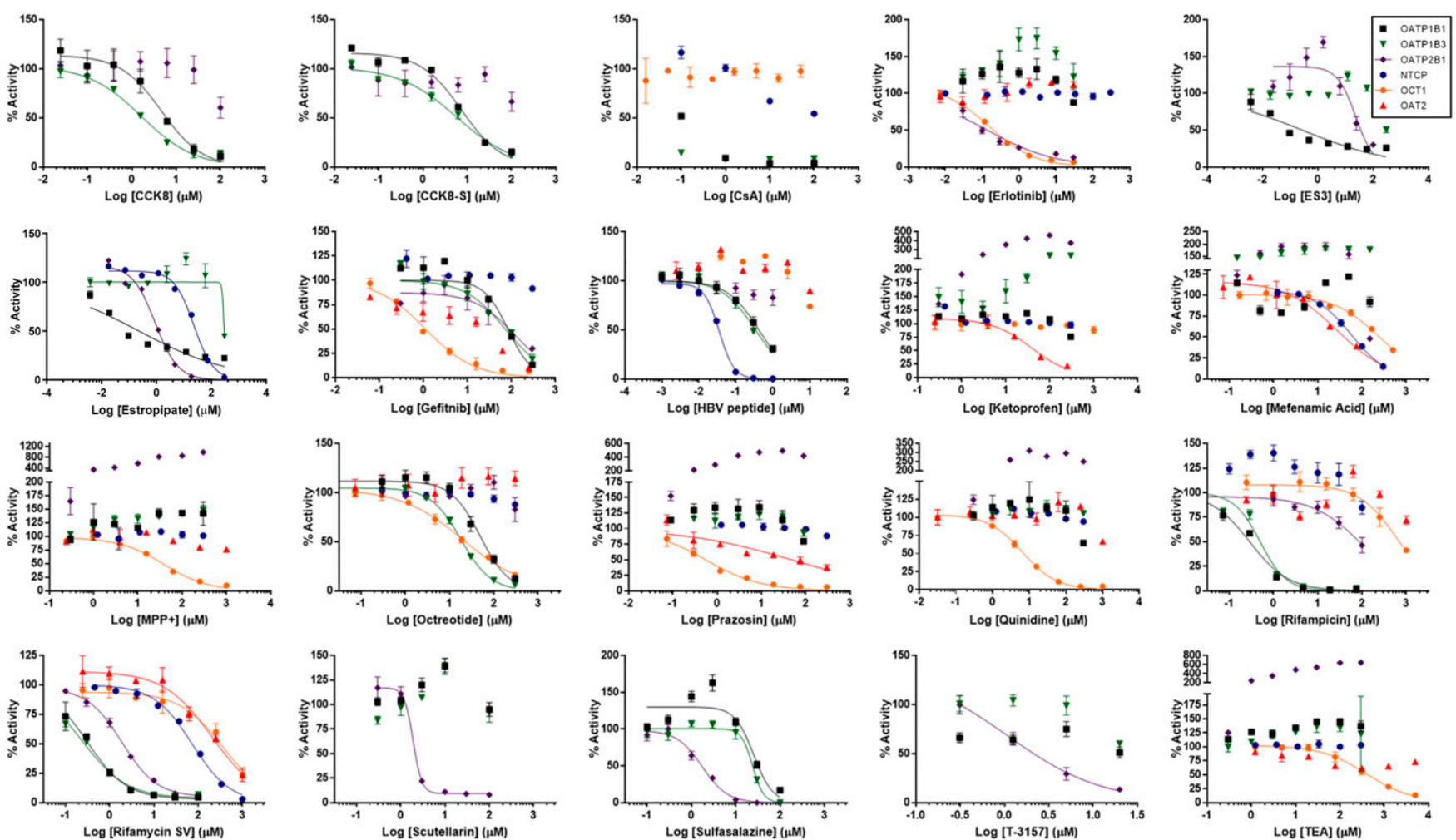

Fig. 1. Concentration-dependent inhibition curves of 20 compounds against six hepatic uptake SLCs, measured using HEK-transfected cells. Data points represent the mean and S.D. of $n=3$. Rosuvastatin was used a probe substrate for OATP1B1/1B3/2B1 inhibition, whereas taurocholate, metformin, and cyclic GMP were used as probes to assess inhibition of NTCP, OCT1, and OAT2, respectively. Concentrations of probe substrates used were below their measured $K_{m}$ values. CsA, cyclosporine A; MPP+, 1-methyl-4-phenylpyridinium.

generally within $25 \%-50 \%$ of the observed values, with the exception of rosuvastatin (Fig. 3; Table 4). Observed pravastatin AUC ratios from the two separate clinical studies spanned a wide range (2.3-4.6), and the scaled $f_{t, O A T P 1 B 1 / 1 B 3}$ well recovered the higher AUC ratio. Notable underprediction of rosuvastatin DDI is plausibly due to lack of consideration of intestinal efflux inhibition by rifampicin in the static model (Kosa et al., 2018; Ufuk et al., 2018). In vitro-in vivo extrapolation of the scaled $f_{t}$ via other transporters or for other substrate drugs could not be evaluated due to lack of relevant clinical data to verify.

\section{Discussion}

Primary human hepatocytes in suspension or plated cultures have become a major tool to characterize transport mechanisms and quantify uptake clearance in support of pharmacokinetics and DDI predictions (Brouwer et al., 2013; Shitara et al., 2013; Zamek-Gliszczynski et al., 2013).
However, due to a lack of known selective inhibitors, the reliable quantification of individual transporter contribution has been challenging. In this study, we screened several inhibitors across six major hepatic sinusoidal uptake transporters, using individually transfected human liver SLCs in HEK293 cells, to identify selective inhibitor conditions that can be used to support SLC-phenotyping with PHHs. It was found that OATPs can be differentiated from other SLCs by incubating hepatocytes with rifamycin SV $(20 \mu \mathrm{M})$, and OATP2B1 can be differentiated from OATP1B1/3 using rifampicin at a lower concentration $(10 \mu \mathrm{M})$. However, we were unable to identify selective OATP1B1 and OATP1B3 inhibitors to delineate their individual contributions. Incubation of HBVpep $(0.1 \mu \mathrm{M})$ with hepatocytes can confirm NTCP function versus other SLCs. Quinidine $(100 \mu \mathrm{M})$ and ketoprofen $(100-300 \mu \mathrm{M})$ can be used to phenotype OCT1 and OAT2, respectively. Finally, rifamycin SV (1 mM) is a pan-SLC inhibitor and can be used to assess passive versus active uptake. The

TABLE 2

Summary of percentage inhibition of individual transporters measured in the transfected HEK293 cells with the inhibitor conditions selected for SLC-phenotyping

\begin{tabular}{lcccrrrr}
\hline \multirow{2}{*}{ Inhibitor Conditions } & \multicolumn{7}{c}{ \% Inhibition in HEK Cells } \\
\cline { 2 - 6 } & OATP1B1 & OATP1B3 & OATP2B1 & NTCP & OCT1 & OAT2 \\
\hline Rifampicin $(10 \mu \mathrm{M})$ & 93 & 96 & 15 & -20 & -9 & 12 & OATP1B1/1B3 vs. OATP2B1 \\
Rifamycin SV $(20 \mu \mathrm{M})$ & 95 & 94 & 89 & 21 & 13 & 4 & OATPs vs. other SLC \\
HBV $(0.1 \mu \mathrm{M})$ & 20 & 25 & 7 & 93 & -17 & -24 & NTCP vs. other SLC \\
Ketoprofen $(100 \mu \mathrm{M})$ & -8 & -132 & -360 & -1 & 6 & 68 & OAT2 vs. other SLC \\
Quinidine $(100 \mu \mathrm{M})$ & -9 & -12 & -196 & 2 & 92 & -18 & OCT1 vs. other SLC \\
Rifamycin SV $(1 \mathrm{mM})$ & 97 & 96 & 95 & 93 & 72 & 75 & Active vs. passive \\
\hline
\end{tabular}


TABLE 3

Substrate affinity of various endogenous compounds and drugs to the six major hepatic uptake transporters

Uptake ratio of $>2$ is used as an arbitrary criterion to suggest substrate affinity.

\begin{tabular}{|c|c|c|c|c|c|c|c|c|}
\hline \multirow{2}{*}{ Substrate } & \multirow{2}{*}{ ECCS class } & \multirow{2}{*}{ Conc. } & \multicolumn{6}{|c|}{ Mean Uptake Ratio (vs. HEK-WT) } \\
\hline & & & OATP1B1 & OATP1B3 & OATP2B1 & NTCP & OCT1 & OAT2 \\
\hline & & $\mu M$ & & & & & & \\
\hline CCK8 & - & 1 & 26 & 371 & 1.0 & 1.6 & 0.5 & 1.5 \\
\hline Bromfenac $^{a}$ & $1 \mathrm{~A}$ & 1 & 2.4 & 0.9 & 0.7 & 0.4 & 0.8 & 4.4 \\
\hline cGMP & $1 \mathrm{~A}$ & 1 & 1.1 & 1.7 & 1.5 & 2.5 & 1.1 & 21.1 \\
\hline Entacapone $e^{a}$ & $1 \mathrm{~A}$ & 1 & 3.6 & 2.1 & 4.8 & 0.6 & 1.0 & 2.1 \\
\hline Fluorescein $^{a}$ & $1 \mathrm{~A}$ & 1 & 3.7 & 7.0 & 1.7 & 1.1 & 1.2 & 1.9 \\
\hline Meloxicam $^{a}$ & $1 \mathrm{~A}$ & 1 & 1.2 & 0.9 & 0.9 & 0.4 & 0.8 & 6.9 \\
\hline $\mathrm{R}$-warfarin & $1 \mathrm{~A}$ & 0.5 & 0.6 & 0.7 & 0.5 & 0.8 & 0.8 & 10.7 \\
\hline S-warfarin & $1 \mathrm{~A}$ & 0.5 & 0.9 & 0.6 & 0.7 & 0.6 & 0.9 & 3.6 \\
\hline Tolbutamide & $1 \mathrm{~A}$ & 1 & 0.7 & 0.9 & 0.5 & 1.1 & 0.9 & 2.6 \\
\hline Fluvastatin & $1 \mathrm{~B}$ & 1 & 2.8 & 2.6 & 4.6 & 3.3 & 0.7 & 0.8 \\
\hline Pitavastatin & $1 \mathrm{~B}$ & 1 & 11.0 & 6.0 & 4.6 & 3.6 & 1.9 & 0.5 \\
\hline E3S & $3 \mathrm{~A}$ & 1 & 58 & 3.6 & 117 & 10 & 0.9 & 2.2 \\
\hline E17G & $3 \mathrm{~B}$ & 1 & 423 & 68 & 0.7 & 0.8 & 1.2 & 1.4 \\
\hline Pravastatin & $3 \mathrm{~B}$ & 1 & 116 & 32.2 & 21 & 3.3 & 1.1 & 0.8 \\
\hline R3G & $3 \mathrm{~B}$ & 1 & 3.4 & 3.6 & 53.9 & 1.4 & 1.0 & 1.6 \\
\hline Rosuvastatin & $3 \mathrm{~B}$ & 1 & 102.3 & 50.7 & 66.2 & 11.8 & 1.8 & 1.2 \\
\hline Taurocholate & $3 \mathrm{~B}$ & 1 & 11 & 26 & 0.8 & 185 & 0.4 & 1.6 \\
\hline Metformin & 4 & 10 & 1.1 & 0.8 & 0.8 & 1.3 & 27.0 & 1.6 \\
\hline Ranitidine & 4 & 1 & 1.0 & 1.1 & 1.0 & 1.0 & 10.1 & 2.4 \\
\hline Thiamine & 4 & 1 & 1.4 & 1.3 & 0.8 & 0.6 & 15.1 & 1.1 \\
\hline
\end{tabular}

Conc., concentration; WT, wild type.

${ }^{a}$ OATP1B1 and OAT2 data reproduced from our previous reports (Kimoto et al., 2018).

described panel of inhibitors could facilitate in vitro characterization of transport mechanisms involved in the hepatic uptake of NMEs, candidate SLC biomarkers, and tool compounds. For example, in the present study, it was possible to confirm that uptake is dominated by individual SLCs $\left(f_{t}>0.8\right)$ for substrates such as CCK8, pravastatin, pitavastatin, and E17G (OATP1B1/3); cGMP, meloxicam, warfarin enantiomers, and tolbutamide (OAT2); and R3G (OATP2B1). A number of compounds, however, are transported by more than one SLC in PHHs (e.g., E3S, entacapone, fluvastatin, rosuvastatin, and ranitidine) (Table 4).

Based on the current study, we propose a stepwise approach to phenotyping hepatic uptake for NMEs and drugs (Fig. 4). First, ECCS, a framework that is based on molecular properties of compounds, can act as a first-tier predictor for the substrate affinity for the hepatic uptake transporter(s). Our previous evaluation of transporter-mediated DDIs per ECCS class validated the predominant role of OATPs in the hepatic clearance of class 1B and 3B drugs (Varma et al., 2015, 2017a; El-Kattan et al., 2016). More recent studies clearly demonstrated OAT2-mediated uptake contribution to the clearance of class 1A drugs (Bi et al., 2018a,b; Kimoto et al., 2018). Additionally, drugs such as sumatriptan, morphine, and ondansetron, which showed association of pharmacokinetic variability with the SLC22A1 genotype, belong to ECCS classes 2 and 4 (Tzvetkov et al., 2013; Matthaei et al., 2016). Therefore, OCT1-mediated hepatic uptake may potentially contribute to the clearance of class 2 and 4 compounds. Evaluating the substrate affinity using single-transfected HEK293 cells or alternative overexpressed cell systems is the second logical step in confirming the potential for individual transporter(s) to contribute to hepatic uptake. Accordingly, for selective substrates, $\mathrm{PHH}$ studies with selective inhibitors and a pan-SLC inhibitor can be used to estimate transporter-specific active uptake and passive clearances. For the substrates of multiple transporters, the proposed inhibition panel in part or full can be used to estimate $f_{t}$ by individual transporters. Finally, correction for expression differences between the in vitro isolated hepatocytes and human liver is warranted to estimate in vivo relevant scaled $f_{t}$.

Incubations at low temperature $\left(4^{\circ} \mathrm{C}\right.$ or on ice) or in the presence of pan-SLC inhibitor (e.g., rifamycin SV) are commonly used for estimating passive uptake (Poirier et al., 2008; Ulvestad et al., 2011; Jones et al., 2012; Nordell et al., 2013; Varma et al., 2014; Bi et al., 2017). Low-temperature incubations can impact membrane diffusion or passive transport and could overestimate active transport rates (Poirier et al., 2008). However, unlike with in vitro models, such as HEK293 and MDCK cells, our previous results suggested similar uptake by $\mathrm{PHHs}$ at $4^{\circ} \mathrm{C}$ and in the presence of $1 \mathrm{mM}$ rifamycin $\mathrm{SV}\left(37^{\circ} \mathrm{C}\right)$ (with a few exceptions), which may be due to cell membrane composition differences between the cell types (Bi et al., 2017). Overall, incubation at $4^{\circ} \mathrm{C}$ may provide a reasonable measure of passive uptake in human hepatocytes; however, follow-up studies may be needed to confirm that the passive diffusion is not reduced due to bilayer rigidity at low temperature. We believe rifamycin $\mathrm{SV}$ at $1 \mathrm{mM}$, where all the major SLCs on the sinusoidal membrane of human hepatocytes are mostly inhibited, can better serve the purpose. Alternatively, a cocktail of selective inhibitors may be used to recover the active and passive $f_{t}$ values.

The relative contributions of sodium-dependent (NTCP) and sodium-independent (e.g., OATPs) uptake processes in hepatocytes are often measured using sodium-free buffers (McRae et al., 2006; Bi et al., 2013). We previously noted that sodium-free buffers (three different buffer compositions) also impacted OATP1B1/1B3-mediated transport in overexpression cell systems (Bi et al., 2017). OATP activity is generally believed to be sodium- and potassium-independent but can be modulated by a change in proton gradient or plasma membrane potential (Jacquemin et al., 1994; Martinez-Becerra 

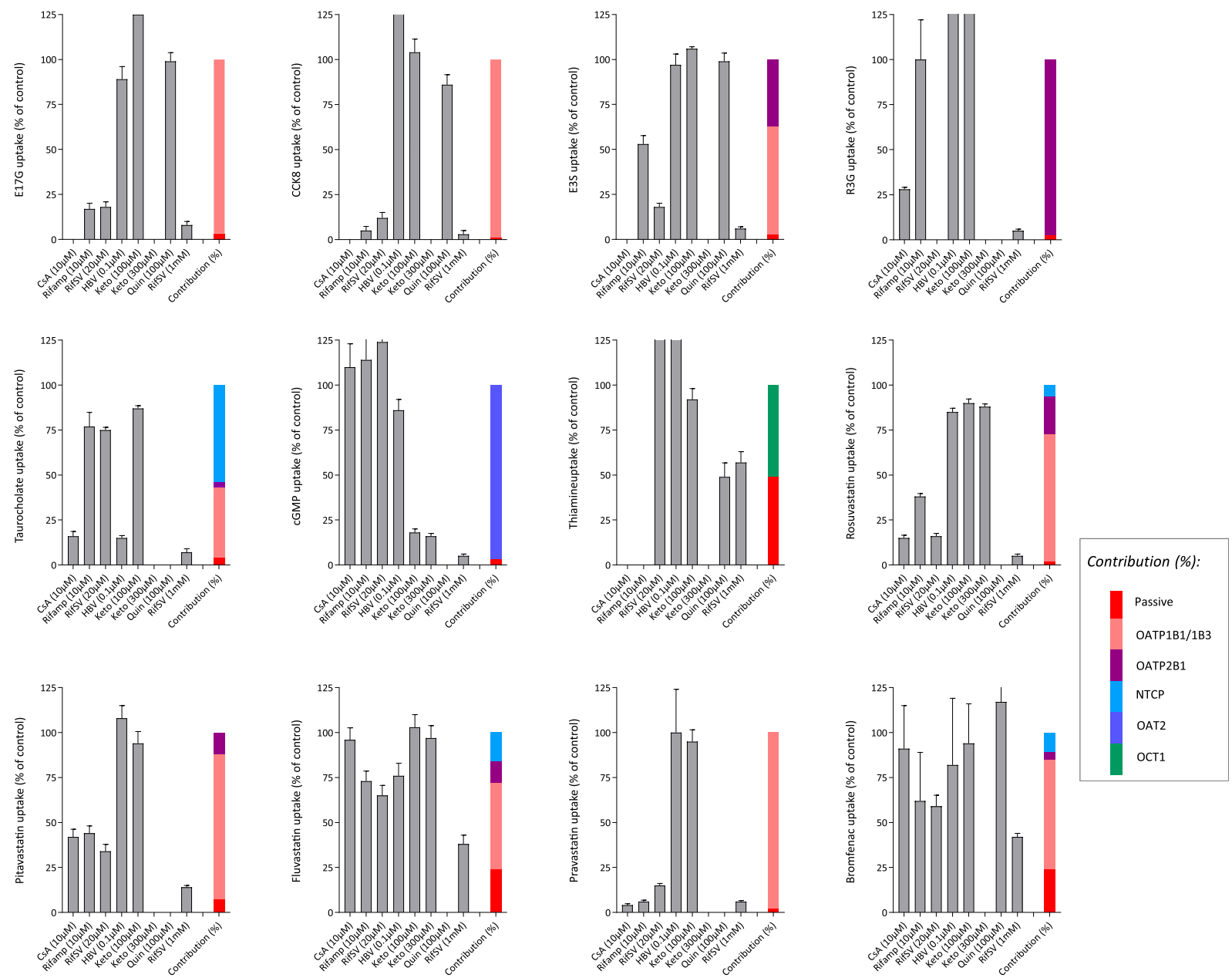

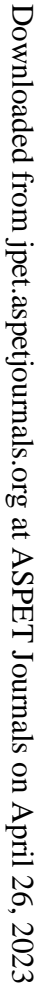
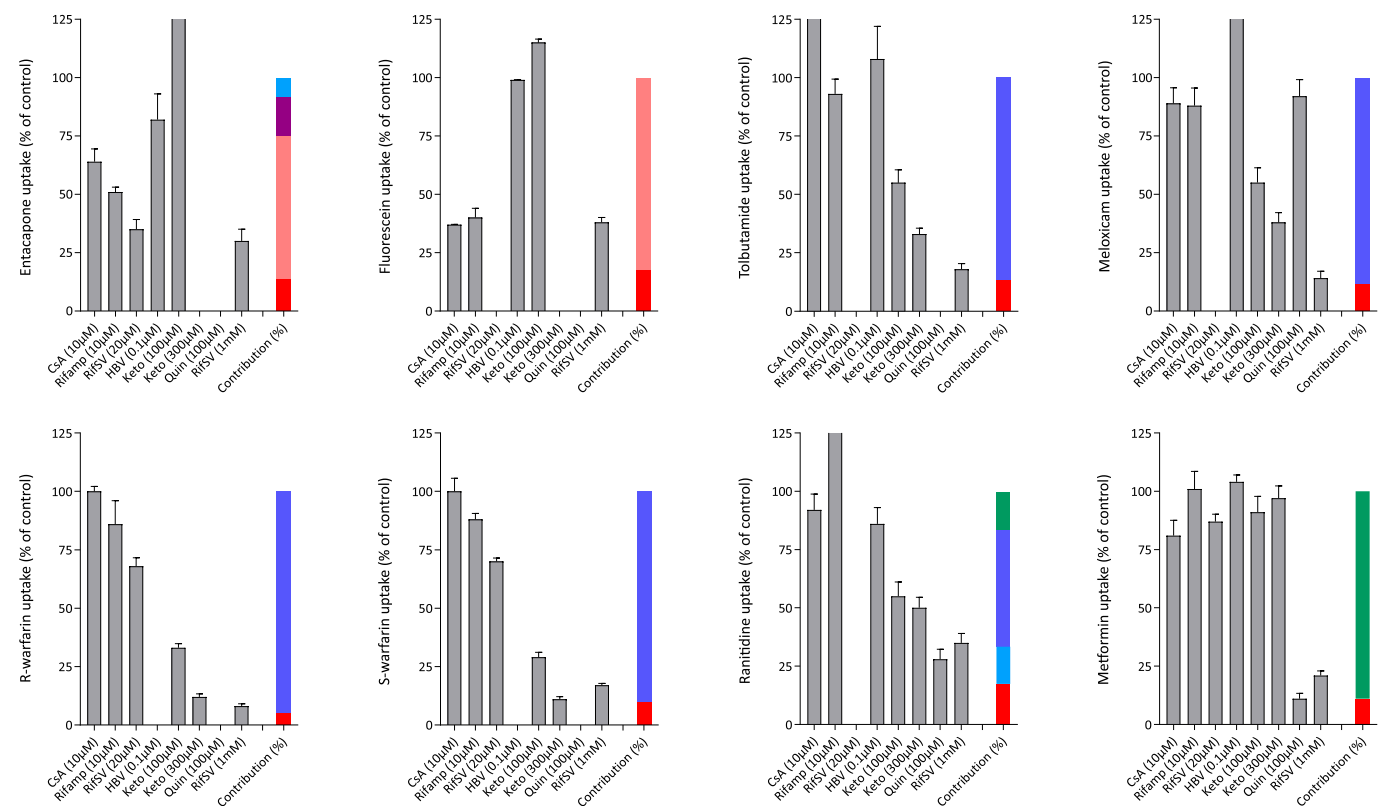

Fig. 2. SLC-phenotyping of 20 probe substrates and drugs in plated human hepatocytes using the proposed panel of transport inhibitors. Gray bars show the effect of inhibitors on total uptake (percentage of control), and multicolor bars depict scaled $f_{t} . N=3$ (mean, S.D.). Percentage contribution was estimated assuming $5 \mu \mathrm{M}$ rifampicin inhibits OATP1B1/1B3 alone, $20 \mu \mathrm{M}$ rifamycin SV inhibits OATP1B1/1B3/2B1, HBV peptide inhibits NTCP alone, ketoprofen inhibits OAT2, quinidine inhibits OCT1, and $1 \mathrm{mM}$ rifamycin SV inhibits all six SLCs. When uptake inhibition was $<15 \%$ (arbitrary cutoff to account for experimental variability) by any one condition, that particular mechanism(s) was assumed to be unaffected (i.e., $0 \%$ inhibition). No bars, conditions not evaluated. Cryopreserved human hepatocyte lot Hu8246 was used. CsA, cyclosporine A. 
TABLE 4

Estimated scaled fraction transport by individual SLCs and passive diffusion, and the predicted statin DDIs with a single dose of rifampicin (600 mg) using scaled $f_{t, O A T P 1 B 1 / 1 B 3}$

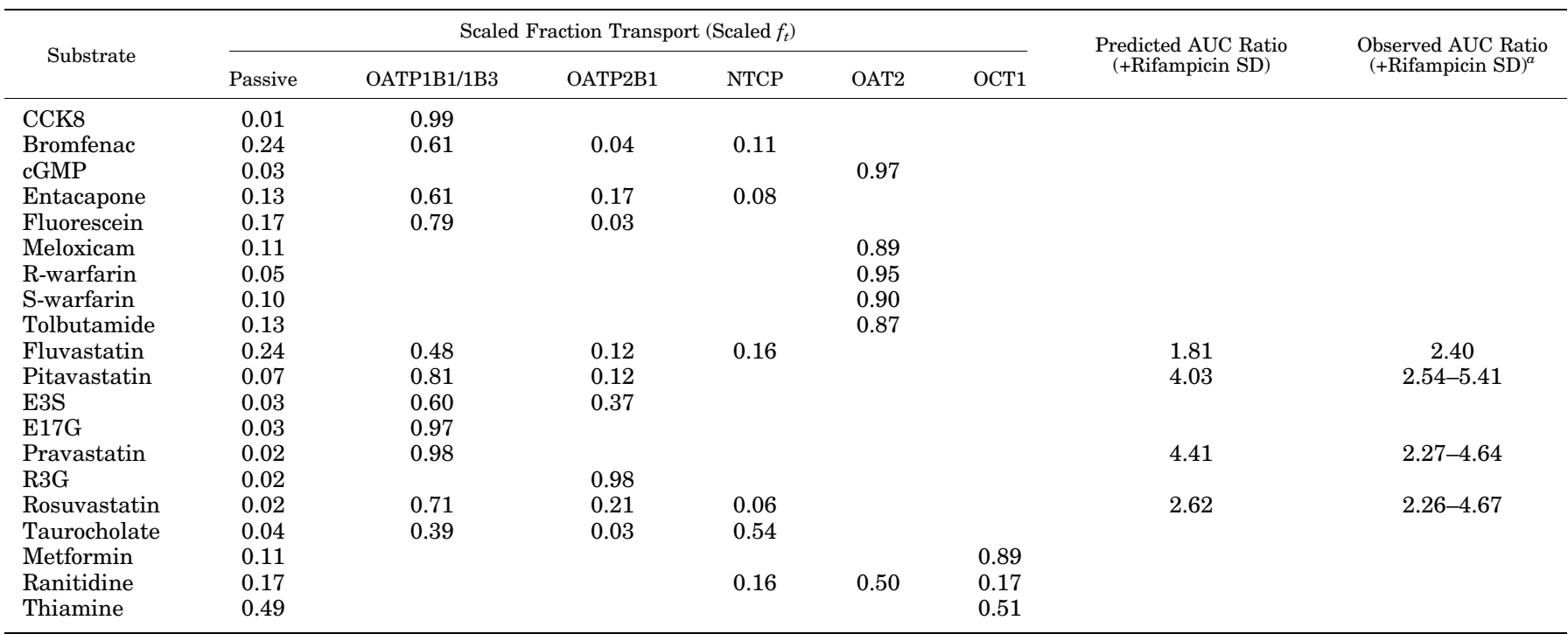

SD, standard deviation.

${ }^{a}$ Range of AUC ratios shown when data from multiple clinical studies were available. References for the observed clinical data are given in Supplemental Table S1.

et al., 2011). Even when the pH of the buffers is monitored, it is possible that the sodium-free buffers alter the membrane potential to a varying degree, leading to an indirect effect on OATP1B1 and OATP1B3. A similar effect of sodium-free ( $N$-methyl-Dglucamine or choline substituted) buffers has been noted for OATP2B1 (Sai et al., 2006). Therefore, HBVpep, which selectively inhibits NTCP at $0.1 \mu \mathrm{M}\left(\mathrm{IC}_{50} \sim 0.04 \mu \mathrm{M}\right)$, is a better alternative to discern the role of NTCP in hepatic uptake.

The six SLCs (OATP1B1, OATP1B3, OATP2B1, NTCP, OAT2, and OCT1) studied here were shown to mediate hepatic uptake of various endogenous chemicals and drugs (Giacomini et al., 2010; Zamek-Gliszczynski et al., 2018). Assignment of $f_{t}$

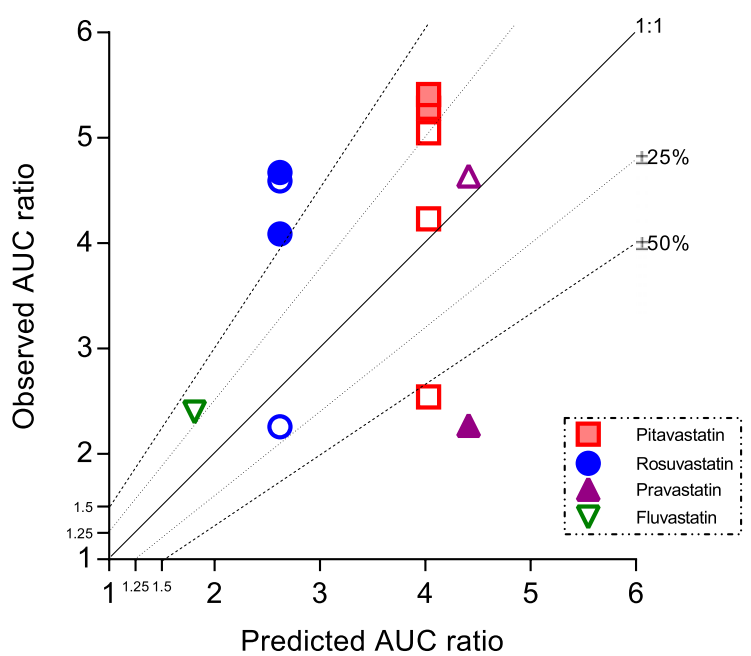

Fig. 3. Predicted versus observed AUC ratio of statins when administrated orally with rifampicin (single dose, $600 \mathrm{mg}$ ). AUC ratio was predicted using the in vitro scaled $f_{t, O A T P 1 B 1 / 1 B 3}$, using a static model (eqs. 5-9). When available, mean AUC ratios from individual clinical studies are presented. Open symbols, statins dosed in micro/small dose in a cocktail/cassette with other probe substrates; closed symbols, statins dosed alone (Supplemental Table S1). Solid line represents unity, and dashed and dotted lines represent $\pm 25 \%$ and $\pm 50 \%$ range, respectively. could be inaccurate in cases where substrate-dependent inhibition is apparent (Izumi et al., 2013) and/or unknown transporters are involved. For example, Izumi et al. (2013) have reported substrate-dependent OATP1B1 inhibition using prototypical substrates, such as E17G, E3S, and sulfobromophthalein. However, the $K_{i}$ values of cyclosporine and rifampicin determined against 12 drug substrates (e.g., statins, sartans, and glinides) have been reported to be within $<55 \%$ coefficient of variance (CV) of the average values, suggesting the substrate dependence is generally minimal with clinically used drug substrates (Izumi et al., 2015). Additionally, OATP1B1-mediated uptake of all substrate drugs is completely inhibited ( $>90 \%$ ) at $10 \mu \mathrm{M}$ rifampicin-inhibitory conditions proposed in the present study. Overall, the potential for substrate dependence, if any, to influence $f_{t}$ estimates in the proposed phenotyping approach appears low. Nevertheless, concentration-dependent inhibition studies using transfected cell systems may be considered to avoid uncertainties in the process of SLC-phenotyping.

Of note, considerable efforts have focused on OCT1 because its expression in human liver is greater $(>10$-fold) than that of OCT3 (SLC22A3) (Nishimura and Naito, 2005; Vildhede et al., 2015). Moreover, a second highly expressed human liver OAT, OAT7 (SLC22A9), was not studied because of its selectivity for steroid sulfates and the lack of selective inhibitors (Mathialagan et al., 2018; Vildhede et al., 2018). These less-studied transporters could be of relevance for specific substrates, and further work is needed to phenotype their contribution.

Fraction transport determined using chemical inhibition in $\mathrm{PHH}$ s was scaled using transporter-specific REF-derived using quantitative proteomics (Kimoto et al., 2012; Qiu et al., 2013; Vildhede et al., 2018). This scaling allowed for the correction of expression differences in the various SLCs between the in vitro systems (PHH) versus liver in vivo. Subsequently, scaled $f_{t}$ values were used to predict DDIs of statins with rifampicin. At clinically relevant plasma unbound 

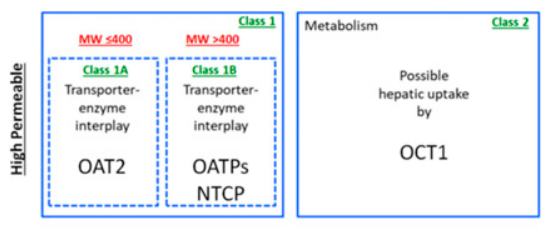

ECCS: Predict

likely transporters

involved in

hepatic uptake
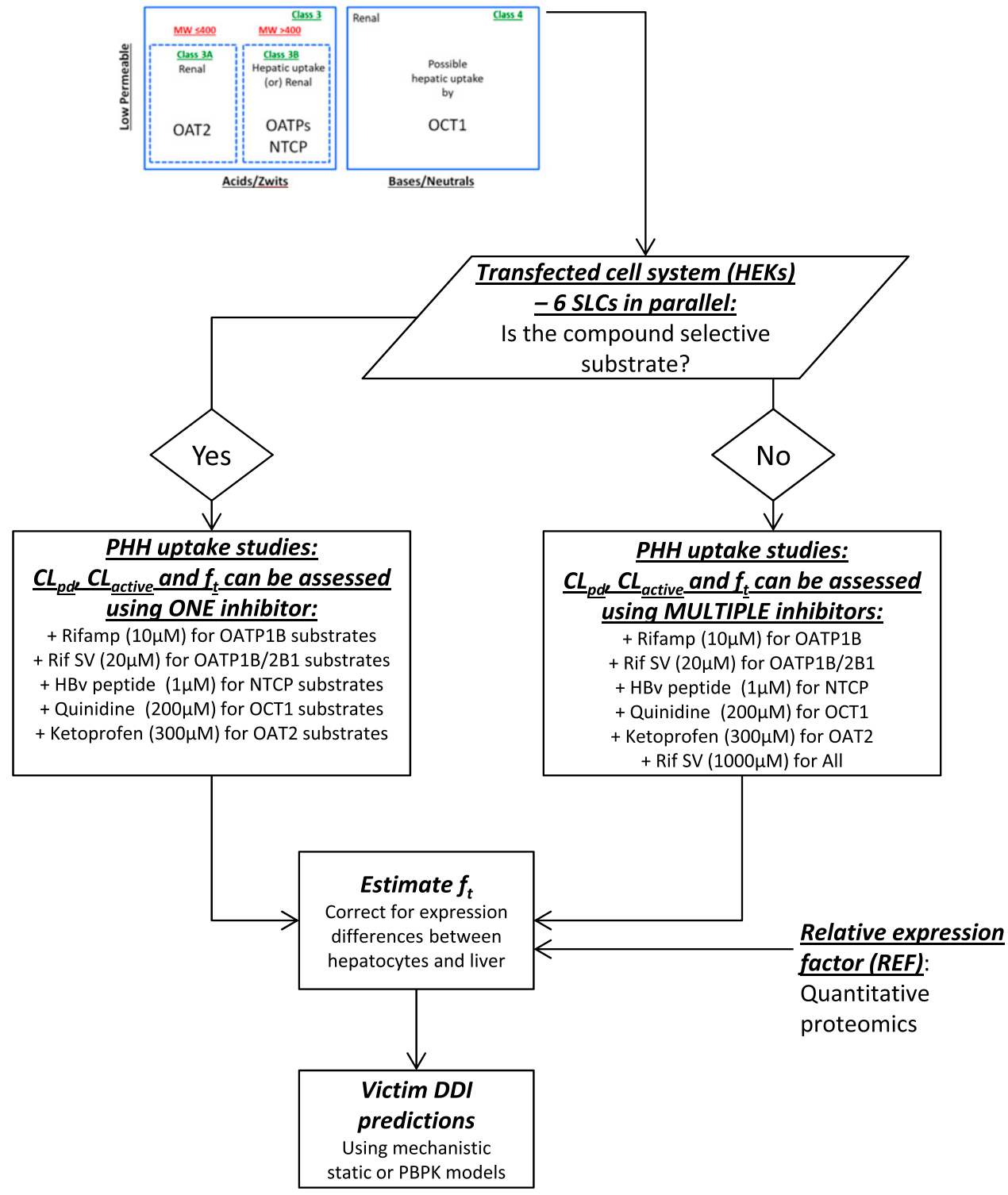

Fig. 4. A pragmatic SLC-phenotyping strategy to discern the role of major transporters in the hepatic uptake of substrate drug and measure the in vitro transporter parameters-passive $\left(\mathrm{CL}_{p d}\right)$ and active $\left(\mathrm{CL}_{\text {active }}\right)$ uptake clearance and the fraction transported by a given SLC $\left(f_{t}\right)$. Initially, ECCS can be used to predict the likely transporters involved in the hepatic uptake. Subsequently, transport studies using overexpressed systems can inform substrate activity. In vitro transport parameters can accordingly be obtained using one specific inhibitor or a battery of selective inhibitor conditions. Fraction transport obtained using a chemical inhibition panel in human hepatocyte incubations needs to be corrected based on relative expression of each transporter in liver tissue versus hepatocytes (REF). The scaled $f_{t}$ can be used to predict victim DDIs via mechanistic static or physiologically based pharmacokinetic (PBPK) models.

concentrations, rifampicin selectively inhibits OATP1B1/1B3mediated hepatic uptake clearance, and therefore, scaled $f_{t, O A T P 1 B 1 / 1 B 3}$ reasonably recovered the AUC ratios of statins, with the exception of rosuvastatin (Fig. 3). The contribution of increased intestinal absorption to the observed AUC ratio, due to inhibition of breast cancer resistance protein (BCRP)-mediated efflux, may explain the notable underprediction for rosuvastatin (Table 4). The $\mathrm{IC}_{50}$ values for human BCRP and P-glycoprotein and the corresponding gut concentration/ $/ \mathrm{IC}_{50}$ ratio indicates that rifampicin can impact the clinical pharmacokinetics of BCRP and P-glycoprotein substrates at the dose (600-mg single dose) recommended to probe OATP1B activity (Kosa et al., 2018). This is consistent with our recent rosuvastatin-rifampicin DDI studies in cynomolgus monkey, where AUC ratios were well predicted following intravenous administration of rosuvastatin but underpredicted following oral dose, particularly at higher doses of rifampicin, suggesting that the disconnect in the IVIV extrapolation of rosuvastatin-rifampicin DDIs is due to a lack of consideration of BCRP-mediated intestinal efflux (Kosa et al., 2018; Ufuk et al., 2018). IVIV extrapolation of the scaled $f_{t}$ via other transporters or for other substrate drugs could not be evaluated due to lack of relevant clinical data.

\section{Conclusions}

In conclusion, we have proposed a transporter phenotyping approach to evaluate the contribution of the major basolateral SLCs to hepatic uptake using primary human hepatocytes. Selective inhibitor conditions were identified by screening 
about 20 inhibitors across six major hepatic SLCs, including OATP1B1/1B3/2B1, NTCP, OAT2, and OCT1. Using these conditions, the scaled $f_{t}$ by individual SLCs was characterized for about 20 different substrates, and an IVIV extrapolation was evaluated where clinical DDI data were available. To our knowledge, this is the first study to systematically evaluate and validate a phenotyping approach to probe activity involving six major human hepatic SLCs.

\section{Authorship Contributions}

Participated in research design: Bi, Costales, Feng, Tremaine, Varma, Rodrigues.

Conducted experiments: Bi, Mathialagan, Eatemadpour, Lazzaro, Tylaska, West, Scialis, Zhang, Umland, Kimoto.

Performed data analysis: Bi, Costales, Varma.

Wrote or contributed to the writing of the manuscript: Bi, Costales, Mathialagan, Scialis, Tess, Tremaine, Rodrigues, Varma.

\section{References}

Bi YA, Lin J, Mathialagan S, Tylaska L, Callegari E, Rodrigues AD, and Varma MVS (2018a) Role of hepatic organic anion transporter 2 in the pharmacokinetics of $\mathrm{R}$ - and S-warfarin: in vitro studies and mechanistic evaluation. Mol Pharm 15: 1284-1295.

Bi YA, Mathialagan S, Tylaska L, Fu M, Keefer J, Vildhede A, Costales C, Rodrigues $\mathrm{AD}$, and Varma MVS (2018b) Organic anion transporter 2 mediates hepatic uptake of tolbutamide, a CYP2C9 probe drug. J Pharmacol Exp Ther 364:390-398.

Bi YA, Qiu X, Rotter CJ, Kimoto E, Piotrowski M, Varma MV, Ei-Kattan AF, and Lai Y (2013) Quantitative assessment of the contribution of sodium-dependent taurocholate co-transporting polypeptide (NTCP) to the hepatic uptake of rosuvastatin, pitavastatin and fluvastatin. Biopharm Drug Dispos 34:452-461.

Bi YA, Scialis RJ, Lazzaro S, Mathialagan S, Kimoto E, Keefer J, Zhang H, Vildhede $\mathrm{AM}$, Costales C, Rodrigues $\mathrm{AD}$, et al. (2017) Reliable rate measurements for active and passive hepatic uptake using plated human hepatocytes. AAPS J 19:787-796. Brouwer KL, Keppler D, Hoffmaster KA, Bow DA, Cheng Y, Lai Y, Palm JE, Stieger B, and Evers R; International Transporter Consortium (2013) In vitro methods to support transporter evaluation in drug discovery and development. Clin Pharmacol Ther 94:95-112.

Chothe PP, Wu S-P, Ye Z, and Hariparsad N (2018) Assessment of transportermediated and passive hepatic uptake clearance using rifamycin-SV as a paninhibitor of active uptake. Mol Pharm 15:4677-4688.

De Bruyn T, Ye Z-W, Peeters A, Sahi J, Baes M, Augustijns PF, and Annaert PP (2011) Determination of OATP-, NTCP- and OCT-mediated substrate uptake activities in individual and pooled batches of cryopreserved human hepatocytes. Eur J Pharm Sci 43:297-307.

El-Kattan AF and Varma MVS (2018) Navigating transporter Sciences in pharmacokinetics characterization using the extended clearance classification system. Drug Metab Dispos 46:729-739.

El-Kattan AF, Varma MV, Steyn SJ, Scott DO, Maurer TS, and Bergman A (2016) Projecting ADME behavior and drug-drug interactions in early discovery and development: application of the extended clearance classification system. Pharm Res 33:3021-3030.

Elmorsi Y, Barber J, and Rostami-Hodjegan A (2016) Ontogeny of hepatic drug transporters and relevance to drugs used in pediatrics. Drug Metab Dispos 44: 992-998.

Giacomini KM, Huang SM, Tweedie DJ, Benet LZ, Brouwer KL, Chu X, Dahlin A, Evers R, Fischer V, Hillgren KM, et al.; International Transporter Consortium (2010) Membrane transporters in drug development. Nat Rev Drug Discov 9 $215-236$.

Izumi S, Nozaki Y, Komori T, Maeda K, Takenaka O, Kusano K, Yoshimura T, Kusuhara H, and Sugiyama Y (2013) Substrate-dependent inhibition of organic anion transporting polypeptide 1B1: comparative analysis with prototypical probe substrates estradiol-17 $\beta$-glucuronide, estrone-3-sulfate, and sulfobromophthalein. Drug Metab Dispos 41:1859-1866.

Izumi S, Nozaki Y, Komori T, Takenaka O, Maeda K, Kusuhara H, and Sugiyama Y (2017) Comparison of the predictability of human hepatic clearance for organic anion transporting polypeptide substrate drugs between different in vitro-in vivo extrapolation approaches. J Pharm Sci 106:2678-2687.

Izumi S, Nozaki Y, Maeda K, Komori T, Takenaka O, Kusuhara H, and Sugiyama Y (2015) Investigation of the impact of substrate selection on in vitro organic anion transporting polypeptide 1B1 inhibition profiles for the prediction of drug-drug interactions. Drug Metab Dispos 43:235-247.

Jacquemin E, Hagenbuch B, Stieger B, Wolkoff AW, and Meier PJ (1994) Expression cloning of a rat liver $\mathrm{Na}(+)$-independent organic anion transporter. Proc Natl Acad Sci USA 91:133-137.

Jones HM, Barton HA, Lai Y, Bi YA, Kimoto E, Kempshall S, Tate SC, El-Kattan A Houston JB, Galetin A, et al. (2012) Mechanistic pharmacokinetic modeling for the prediction of transporter-mediated disposition in humans from sandwich culture human hepatocyte data. Drug Metab Dispos 40:1007-1017.

Karlgren M, Vildhede A, Norinder U, Wisniewski JR, Kimoto E, Lai Y, Haglund U, and Artursson P (2012) Classification of inhibitors of hepatic organic anion transporting polypeptides (OATPs): influence of protein expression on drug-drug interactions. J Med Chem 55:4740-4763.
Kato M, Chiba K, Hisaka A, Ishigami M, Kayama M, Mizuno N, Nagata Y, Takakuwa S, Tsukamoto Y, Ueda K, et al. (2003) The intestinal first-pass metabolism of substrates of CYP3A4 and P-glycoprotein-quantitative analysis based on information from the literature. Drug Metab Pharmacokinet 18:365-372.

Kimoto E, Mathialagan S, Tylaska L, Niosi M, Lin J, Carlo AA, Tess DA, and Varma MVS (2018) Organic anion transporter 2-mediated hepatic uptake contributes to the clearance of high-permeability-low-molecular-weight acid and zwitterion drugs: evaluation using 25 drugs. J Pharmacol Exp Ther 367:322-334.

Kimoto E, Yoshida K, Balogh LM, Bi YA, Maeda K, El-Kattan A, Sugiyama Y, and Lai Y (2012) Characterization of organic anion transporting polypeptide (OATP) expression and its functional contribution to the uptake of substrates in human hepatocytes. Mol Pharm 9:3535-3542.

König A, Döring B, Mohr C, Geipel A, Geyer J, and Glebe D (2014) Kinetics of the bile acid transporter and hepatitis $\mathrm{B}$ virus receptor $\mathrm{Na}+$ /taurocholate cotransporting polypeptide (NTCP) in hepatocytes. J Hepatol 61:867-875.

König J, Müller F, and Fromm MF (2013) Transporters and drug-drug interactions: important determinants of drug disposition and effects. Pharmacol Rev 65: 944-966.

Kosa RE, Lazzaro S, Bi YA, Tierney B, Gates D, Modi S, Costales C, Rodrigues AD, Tremaine LM, and Varma MV (2018) Simultaneous assessment of transportermediated drug-drug interactions using a probe drug cocktail in cynomolgus monkey. Drug Metab Dispos 46:1179-1189.

Kunze A, Huwyler J, Camenisch G, and Poller B (2014) Prediction of organic aniontransporting polypeptide 1B1- and 1B3-mediated hepatic uptake of statins based on transporter protein expression and activity data. Drug Metab Dispos 42:1514-1521.

Li R, Barton HA, and Varma MV (2014) Prediction of pharmacokinetics and drugdrug interactions when hepatic transporters are involved. Clin Pharmacokinet 53: 659-678.

Martinez-Becerra P, Briz O, Romero MR, Macias RI, Perez MJ, Sancho-Mateo C, Lostao MP, Fernandez-Abalos JM, and Marin JJ (2011) Further characterization of the electrogenicity and $\mathrm{pH}$ sensitivity of the human organic anion-transporting polypeptides OATP1B1 and OATP1B3. Mol Pharmacol 79:596-607.

Mathialagan S, Costales C, Tylaska L, Kimoto E, Vildhede A, Johnson J, Johnson N, Sarashina T, Hashizume K, Isringhausen CD, et al. (2018) In vitro studies with two human organic anion transporters: OAT2 and OAT7. Xenobiotica 48:1037-1049.

Matthaei J, Kuron D, Faltraco F, Knoch T, Dos Santos Pereira JN, Abu Abed M, Prukop T, Brockmöller J, and Tzvetkov MV (2016) OCT1 mediates hepatic uptake of sumatriptan and loss-of-function OCT1 polymorphisms affect sumatriptan pharmacokinetics. Clin Pharmacol Ther 99:633-641.

McRae MP, Lowe CM, Tian X, Bourdet DL, Ho RH, Leake BF, Kim RB, Brouwer KL, and Kashuba $\mathrm{AD}$ (2006) Ritonavir, saquinavir, and efavirenz, but not nevirapine, inhibit bile acid transport in human and rat hepatocytes. J Pharmacol Exp Ther 318:1068-1075.

Mitra P, Weinheimer S, Michalewicz M, and Taub ME (2018) Prediction and quantification of hepatic transporter-mediated uptake of pitavastatin utilizing a combination of the Relative Activity Factor approach and mechanistic modeling. Drug Metab Dispos 46:953-963.

Nishimura M and Naito S (2005) Tissue-specific mRNA expression profiles of human ATP-binding cassette and solute carrier transporter superfamilies. Drug Metab Pharmacokinet 20(6):452-477.

Nordell P, Winiwarter S, and Hilgendorf C (2013) Resolving the distributionmetabolism interplay of eight OATP substrates in the standard clearance assay with suspended human cryopreserved hepatocytes. Mol Pharm 10:4443-4451.

Poirier A, Lavé T, Portmann R, Brun M-E, Senner F, Kansy M, Grimm H-P, and Funk C (2008) Design, data analysis, and simulation of in vitro drug transport kinetic experiments using a mechanistic in vitro model. Drug Metab Dispos 36: $2434-2444$

Qiu X, Bi Y-A, Balogh LM, and Lai Y (2013) Absolute measurement of species differences in sodium taurocholate cotransporting polypeptide (NTCP/Ntcp) and its modulation in cultured hepatocytes. J Pharm Sci 102:3252-3263.

Sai Y, Kaneko Y, Ito S, Mitsuoka K, Kato Y, Tamai I, Artursson P, and Tsuji A (2006) Predominant contribution of organic anion transporting polypeptide OATP-B (OATP2B1) to apical uptake of estrone-3-sulfate by human intestinal Caco-2 cells. Drug Metab Dispos 34:1423-1431.

Shitara Y, Horie T, and Sugiyama Y (2006) Transporters as a determinant of drug clearance and tissue distribution. Eur J Pharm Sci 27:425-446.

Shitara Y, Maeda K, Ikejiri K, Yoshida K, Horie T, and Sugiyama Y (2013) Clinical significance of organic anion transporting polypeptides (OATPs) in drug disposition: their roles in hepatic clearance and intestinal absorption. Biopharm Drug Dispos 34:45-78.

Tzvetkov MV, dos Santos Pereira JN, Meineke I, Saadatmand AR, Stingl JC, and Brockmöller J (2013) Morphine is a substrate of the organic cation transporter OCT1 and polymorphisms in OCT1 gene affect morphine pharmacokinetics after codeine administration. Biochem Pharmacol 86:666-678.

Ufuk A, Kosa RE, Gao H, Bi YA, Modi S, Gates D, Rodrigues AD, Tremaine LM, Varma MVS, Houston JB, et al. (2018) In vitro-in vivo extrapolation of OATP1Bmediated drug-drug interactions in cynomolgus monkey. J Pharmacol Exp Ther 365:688-699.

Ulvestad M, Björquist P, Molden E, Asberg A, and Andersson TB (2011) OATP1B1/ 1B3 activity in plated primary human hepatocytes over time in culture. Biochem Pharmacol 82:1219-1226.

Varma MV, Bi YA, Kimoto E, and Lin J (2014) Quantitative prediction of transporterand enzyme-mediated clinical drug-drug interactions of organic anion-transporting polypeptide 1B1 substrates using a mechanistic net-effect model. J Pharmacol Exp Ther 351:214-223.

Varma MV and El-Kattan AF (2016) Transporter-enzyme interplay: deconvoluting effects of hepatic transporters and enzymes on drug disposition using static and dynamic mechanistic models. J Clin Pharmacol 56 (Suppl 7):S99-S109.

Varma MV, El-Kattan AF, Feng B, Steyn SJ, Maurer TS, Scott DO, Rodrigues AD, and Tremaine LM (2017a) Extended Clearance Classification System (ECCS) 
informed approach for evaluating investigational drugs as substrates of drug transporters. Clin Pharmacol Ther 102:33-36.

Varma MV, Lai Y, and El-Kattan AF (2017b) Molecular properties associated with transporter-mediated drug disposition. Adv Drug Deliv Rev 116:92-99.

Varma MV, Lai Y, Feng B, Litchfield J, Goosen TC, and Bergman A (2012) Physiologically based modeling of pravastatin transporter-mediated hepatobiliary disposition and drug-drug interactions. Pharm Res 29:2860-2873.

Varma MV, Steyn SJ, Allerton C, and El-Kattan AF (2015) Predicting clearance mechanism in drug discovery: extended clearance classification system (ECCS). Pharm Res 32:3785-3802.

Vildhede A, Wiśniewski JR, Norén A, Karlgren M, and Artursson P (2015) Comparative proteomic analysis of human liver tissue and isolated hepatocytes with a focus on proteins determining drug exposure. J Proteome Res 14(8):3305-3314.

Vildhede A, Kimoto E, Rodrigues AD, and Varma MVS (2018) Quantification of hepatic organic anion transport proteins OAT2 and OAT7 in human liver tissue and primary hepatocytes. Mol Pharm 15:3227-3235.

Williams JA, Bauman J, Cai H, Conlon K, Hansel S, Hurst S, Sadagopan N Tugnait M, Zhang L, and Sahi J (2005) In vitro ADME phenotyping in drug discovery: current challenges and future solutions. Curr Opin Drug Discov Dev 8:78-88.

Williamson B, Soars AC, Owen A, White P, Riley RJ, and Soars MG (2013) Dissecting the relative contribution of OATP1B1-mediated uptake of xenobiotics into human hepatocytes using siRNA. Xenobiotica 43:920-931.
Yan H, Peng B, Liu Y, Xu G, He W, Ren B, Jing Z, Sui J, and Li W (2014) Viral entry of hepatitis $\mathrm{B}$ and $\mathrm{D}$ viruses and bile salts transportation share common molecular determinants on sodium taurocholate cotransporting polypeptide. $J$ Virol 88: $3273-3284$

Zamek-Gliszczynski MJ, Lee CA, Poirier A, Bentz J, Chu X, Ellens H, Ishikawa T, Jamei M, Kalvass JC, Nagar S, et al.; International Transporter Consortium (2013) ITC recommendations for transporter kinetic parameter estimation and translational modeling of transport-mediated PK and DDIs in humans. Clin Pharmacol Ther 94:64-79.

Zamek-Gliszczynski MJ, Taub ME, Chothe PP, Chu X, Giacomini KM, Kim RB, Ray AS, Stocker SL, Unadkat JD, Wittwer MB, et al.; International Transporter Consortium (2018) Transporters in drug development: 2018 ITC recommendations for transporters of emerging clinical importance. Clin Pharmacol Ther 104: 890-899.

Zhou F, Zhu L, Wang K, and Murray M (2017) Recent advance in the pharmacogenomics of human Solute Carrier Transporters (SLCs) in drug disposition. Adv Drug Deliv Rev 116:21-36.

Address correspondence to: Manthena V. S. Varma, ADME Sciences, Medicine Design, Worldwide Research and Development, MS 8220-2451, Pfizer Inc., Groton, CT 06340. E-mail: manthena.v.varma@pfizer.com 